$\mathrm{p}<0.005)$ and having a co-infection (10.35[4. 32-25.30]; $\mathrm{p}<0.001)$ remained significant.

Conclusion Having an STI co-infection with MG was the strongest indicator of likelihood of having macrolide resistance which was also associated with being in particular risk groups. These findings are suggestive that macrolide resistance may be maintained in discreet sexual networks that are themselves exposed to antibiotic selection pressures.

Disclosure No significant relationships.

\section{P615 CLINICAL IMPROVEMENT AFTER STANDARD TREATMENT FOR URETHRITIS: THE ROLE OF MYCOPLASMA GENITALIUM}

${ }^{1}$ Clarissa Vergunst* ${ }^{\star}{ }^{2}$ Maarten Schim Van Der Loeff, ${ }^{1}$ Martijn Van Rooijen, ${ }^{3}$ Henry De Vries, ${ }^{2}$ Sylvia Bruisten, ${ }^{4}$ Alje Van Dam. ${ }^{1}$ Public Health Service of Amsterdam, Infectious Diseases, Amsterdam, Netherlands; ${ }^{2}$ Public Health Service Amsterdam, Amsterdam University Medical Center (UMC), Infectious Diseases, Infection and Immunity (AI and II), Amsterdam, Netherlands; ${ }^{3}$ Public Health Service Amsterdam, Amsterdam University Medical Center (UMC), National Institute of Public Health and the Environment (RIVM), Infectious Diseases, Infection and Immunity Institute (AI and II), Epidemiology and Surveillance Unit, Amsterdam, Netherlands; ${ }^{4}$ Municipal Public Health Service Amsterdam, Public Health Laboratory, Amsterdam, Netherlands

10.1136/sextrans-2019-sti.683

Background Mycoplasma genitalium (MG) is a sexually transmitted organism associated with urethritis in men. We examined clinical improvement of symptoms in men treated syndromically for urethritis, and correlated the clinical outcome to MG positivity.

Methods At the STI clinic in Amsterdam, the Netherlands, urethritis is defined as the presence of $\geq 10$ leucocytes per high power field in Gram stains of urethral discharge. The additional presence of intracellular gram-negative diplococci defines gonococcal urethritis. Point-of-care standard therapy for gonococcal urethritis is $1000 \mathrm{mg}$ ceftriaxone and for nongonococcal urethritis is azithromycin $1000 \mathrm{mg}$. From May 2018 onwards, urine samples of all men with urethritis were tested for presence of N. gonorrhoeae (NG), C. trachomatis (CT), and M. genitalium (MG) using TMA assays (Aptima, Hologic). These men were sent a text message two weeks after receiving standard therapy, with a questionnaire about improvement (including resolution) of their urethritis symptoms. We analyzed clinical improvement by MG status.

Results From May through December 2018, 1015 men presented with 1111 episodes of urethritis. Of 88 episodes, there were no results for MG. Of the remaining 1023 episodes, men responded to the text message in 379 cases (37\%). Of 379 cases $87(23 \%)$ were positive for NG, 119 (31\%) for CT, and $81(21 \%)$ for MG. Clinical improvement was reported in 312 episodes (82\%); this was $89 \%$ in NG cases; $82 \%$ in CT cases, and $72 \%$ in MG cases. Clinical improvement was reported by $92 \%(55 / 60), 85 \%(83 / 98)$ and $70 \%(35 /$ $50)$ of those with single infection with $\mathrm{NG}$, CT or MG respectively $(\mathrm{P}=0.009)$; and by $80 \%(110 / 134)$ of those with none of these infections. Those with $\mathrm{MG} / \mathrm{CT}$ co-infection had worse outcomes than those without MG $(\mathrm{P}=0.015)$.

Conclusion Among men with urethritis 82\% improved after standard syndromic treatment. Those with MG/CT co-infection and those with MG single infection had significantly worse treatment results.

Disclosure No significant relationships.

\section{P616 PREDICTIVE MACROLIDE AND FLUOROQUINOLONE RESISTANCE MARKERS IN MYCOPLASMA GENITALIUM FROM THE UK AND IRELAND}

Michaela Day*, Michelle Cole, Hemanti Patel, Helen Fifer, Neil Woodford, Rachel Pitt. Public Health England, National Infection Service, London, UK

\subsection{6/sextrans-2019-sti.684}

Background This study sought to assess the prevalence of macrolide and fluoroquinolone resistance in Mycoplasma genitalium-positive specimens received in PHE's national reference laboratory.

Methods M. genitalium-positive clinical specimens submitted from 59 laboratories across the UK and Ireland were tested for molecular markers of macrolide and fluoroquinolone resistance. The $23 \mathrm{~S}$ rRNA gene and the quinolone-resistance-determining region (QRDR) of parC were amplified by PCR and Sanger sequenced. Single nucleotide polymorphisms (SNPs) associated with clinical treatment failure were detected through sequence alignment in BioNumerics software (V.6.1, Applied Maths, USA).

Results Four hundred and fifty-eight $M$. genitalium-positive specimens were received between 01/09/17 and 28/11/2018 . Sequencing results were available for both gene targets in $389 /$ 458 (84.9\%) specimens. Seventy-one percent (275/389) were predicted to be resistant to macrolides. $23 \mathrm{~S}$ rRNA SNPs detected were A2058G (136/275, 49.5\%), A2059G (131/275, 47.6\%), A2058T (5/275, 1.8\%) and A2059C (3/275, 1.1\%). Eight percent $(31 / 389)$ were predicted to be resistant to fluoroquinolones. parC mutations detected were D87N (16/31, 51.6\%), S83I (12/31, 38.7\%), D87Y (2/31, 6.5\%) and S83R $(1 / 31,3.2 \%)$. Seven percent $(26 / 389)$ were predicted to be resistant to both antimicrobial classes. Only $28 \%$ of positive samples tested were predicted to be susceptible to both classes of antimicrobial.

Conclusion Resistance to macrolides, the current first-line treatment for $M$. genitalium, in specimens received at $\mathrm{PHE}$ from patients attending STI clinics in the UK and Ireland is very high, at $85 \%$. Conversely, resistance to the second-line treatment, moxifloxacin, in these specimens was estimated at $8 \%$ although the actual rate of resistance may be higher as there are many mutations with unknown treatment outcomes. Isolates exhibiting resistance to both antimicrobial classes are of significant public health concern as further treatment options for this organism are limited. Effective surveillance of SNPs in this organism is imperative to further understand the affect on clinical outcome.

Disclosure No significant relationships. 